\title{
Limitations of Standard Immunosuppressive Treatment in ANCA-Associated Vasculitis and Lupus Nephritis
}

\author{
Vladimir Tesar Zdenka Hruskova \\ Department of Nephrology, 1st Faculty of Medicine, General University Hospital, Charles University, Prague, Czech Republic
}

\section{Key Words}

ANCA-associated vasculitis - Cyclophosphamide .

Lupus nephritis

\begin{abstract}
Introduction of the standard immunosuppressive treatment has dramatically changed the outcome of patients with both ANCA-associated vasculitis and lupus nephritis, transforming them from incurable diseases with very high short-term mortality to chronic debilitating diseases with much lower short-term, but still relatively high long-term, morbidity/ mortality. Long-term morbidity with damage accumulating partly due to the adverse events of the available treatment (namely gonadal toxicity, malignancy, bone disease, cataracts, diabetes, and thromboembolic and cardiovascular disease) has become a major concern. Although cyclophosphamide-based regimens have been partly replaced by newer agents in both ANCA-associated vasculitis and lupus nephritis (namely rituximab or mycophenolate, respectively) their short-term and medium-term adverse events may not be significantly less frequent and we can only hope that new treatments will translate into better long-term outcomes including better long-term safety.
\end{abstract}

(c) 2014 S. Karger AG, Basel

$1660-2110 / 14 / 1284-0205 \$ 39.50 / 0$

\section{Introduction}

Corticosteroids and less often other immunosuppressive drugs are used in the treatment of many immunologically mediated glomerular diseases. Adverse events associated with long-term (or repeated) corticosteroid treatment are frequent and may be serious, for example in patients with corticosteroid-dependent minimal change disease or focal segmental glomerulosclerosis.

In this review, however, we will concentrate only on ANCA-associated vasculitis (AAV) and lupus nephritis (LN) as the treatment of these diseases is more standardized and due to their tendency to relapse, long-term maintenance and repeated induction treatment resulting in higher cumulative doses of corticosteroids and cyclophosphamide are frequently necessary. We will briefly review only more traditional (standard) immunosuppressive treatment as specific chapters in this issue are dedicated to the treatment of both AAV and LN with

Biologic Treatment in Glomerular Disease D. Jayne, Cambridge

V. Tesar, Prague

\section{KARGER}

E-Mail karger@karger.com www.karger.com/nec
Vladimir Tesar

Department of Nephrology, General University Hospital

$\mathrm{U}$ nemocnice 2

CZ-128 08 Prague 2 (Czech Republic)

E-Mail vladimir.tesar@vfn.cz 
biologic drugs and will concentrate on the adverse events related to corticosteroid and cyclophosphamide treatment.

New modes of treatment are needed especially in patients refractory, intolerant to, or frequently relapsing on the standard immunosuppressive treatment, namely to avoid or at least lessen the still unacceptably high shortand long-term toxicity of the treatment. Organ damage (e.g. advanced chronic kidney disease), either due to late diagnosis and referral (in AAV), or due to relatively slow response to treatment (in LN) further increases the adverse events of the treatment.

\section{Standard Treatment of AAV: Outcome versus Toxicity of Treatment}

The long-term outcome of patients with AAV has dramatically improved with the introduction of standard immunosuppressive treatment. The median survival of untreated patients with granulomatosis with polyangiitis (GPA; formerly known as Wegener's granulomatosis) was only 5 months (most patients dying of respiratory or renal failure), with 1-year mortality of $82 \%$ and 2 -year mortality of $90 \%$ [1]. Whereas the treatment with corticosteroids in monotherapy only slightly prolonged the median survival to 12.5 months, with most patients dying of infections or the progression of the disease [2], introduction of cyclophosphamide substantially changed the outcome of the patients, and the 1-year mortality decreased to only $20 \%$ [3].

At the beginning of the 1990s most patients with AAV (even with relatively mild disease) were treated for at least 1 year with cyclophosphamide (and initially high-dose and gradually tapered corticosteroids), and then for an undefined period of time with azathioprine and low-dose corticosteroids. In an analysis of 158 patients with GPA (mostly with ENT and lung involvement, only 20\% with the involvement of the kidney [4]) treated with a very high cumulative dose ( $>50 \mathrm{~g}$ ) of cyclophosphamide, $91 \%$ of patients significantly improved and $75 \%$ developed complete remission, but mortality was relatively high at $13 \%$ (considering the absence of generalized disease in about $50 \%$ of patients) and the 15 -year risk of bladder cancer was also very high (16\%) [5]. As oral cyclophosphamide was used for a very long time and repeated with each relapse of the disease, the cumulative dose of cyclophosphamide was usually very high and the patients had a high long-term risk of myelodysplasia and secondary malignancies [6].
Introduction of the induction treatment with highdose corticosteroids and oral cyclophosphamide thus transformed AAV from the acute life-threatening disease with a mean survival of only about 6 months to the chronic disease with slowly accumulated organ damage both due to the activity of the disease and the adverse events of the rather toxic treatment, resulting in a high remission rate of 75-93\% but still unfavorable 5-year survival of 69$91 \%$ in patients with GPA and only $45-76 \%$ in microscopic polyangiitis [7].

A recent retrospective analysis of 445 patients with GPA treated in one large German academic center over four decades clearly demonstrated improving outcome of the patients [8], characterized by decreasing standardized mortality ratio (from 2.1 before 1993 to 1.03 after 1999) with a decreasing proportion of death related to either the activity of the disease or its treatment (from 86.4 to $50 \%$ ) and decreasing relapse rate (from 63.9 to $35.3 \%$ ) despite similar presenting organ manifestations. This improvement could possibly be due to better awareness and earlier diagnosis (due to the availability of ANCA testing) and more prudent use of immunosuppression. Although $80 \%$ of more recent patients are still treated with cyclophosphamide, the cumulative dose of cyclophosphamide has decreased from 67 to $24 \mathrm{~g}$.

Recently reported outcomes of 535 European patients (median age: 60.7 years, $53 \%$ GPA, median creatinine level: $203 \mu \mathrm{mol} / 1$, median follow-up: 5.2 years [9]) confirmed, however, a still relatively high standardized mortality ratio in patients with AAV (compared to age- and sexmatched population) of 2.6 , with infections (48\%) and active vasculitis (19\%) being the main causes of death in the first year, followed by cardiovascular disease $(26 \%)$, malignancy (22\%), and infection (20\%) after the first year.

\section{Minimizing Exposure to Cyclophosphamide and High-Dose Corticosteroids in AAV}

In the last 20 years, studies organized by the European Vasculitis Study Group (EUVAS) have been dedicated to the optimization of the available treatment options with the aim to minimize the exposure to cyclophosphamide [10]. To pinpoint the treatment to the severity of the disease, patients were subdivided into 5 groups (localized, early systemic, generalized, severe, and refractory disease).

It has been demonstrated in patients with generalized AAV (serum creatinine $<500 \mu \mathrm{mol} / \mathrm{l}$ ) that an early compared to later (3 months vs. 12 months) switch from cyclophosphamide to azathioprine does not increase the 
(early) relapse rate (within 18 months; CYCAZAREM study [11]) and that oral continuous cyclophosphamide can be safely replaced, in terms of time to remission and remission rate, by intravenous cyclophosphamide pulses with a much lower cumulative dose of cyclophosphamide (8.2 vs. $15.9 \mathrm{~g}$ ) and lower rate of leukopenia (CYCLOPS study [12]).

However, the long-term outcome of patients treated with a lower cumulative induction dose of cyclophosphamide remains uncertain. The 5-year risk of AAV relapse requiring repeated courses of induction treatment (with a putative increase of cumulative dose of both corticosteroids and cyclophosphamide) still remains about $50 \%$ [7], and is higher in patients with anti-PR3 antibodies and better preserved renal function [13], and may be related to lower initial dose of cyclophosphamide and corticosteroids [14]. In the CYCLOPS study, after a median follow-up of 4.3 years patient and renal survival was the same in both limbs despite an almost doubled risk of relapses in patients treated initially with pulsed cyclophosphamide [15]. In the CYCAZAREM study, after a median follow-up of 8.5 years there was a nonsignificant trend to worse outcome (in terms of relapse rate and ESRD) in patients who were switched early to azathioprine [16]. Similarly, in the NORAM study replacement of cyclophosphamide with methotrexate in the induction treatment in early systemic AAV resulted in a tendency during long-term follow-up to shorter relapse-free survival and longer treatment with corticosteroids and other immunosuppressants (azathioprine, methotrexate, and/or mycophenolate) in patients who were initially treated with methotrexate [17].

Therefore, the strategy aimed at minimizing early exposure to cyclophosphamide may be offset by a higher relapse rate resulting in similar overall cyclophosphamide exposure with limited, if any, positive effect on the long-term outcome of the patients. Newer cyclophosphamide-free therapeutic regimens, e.g. rituximab, may not have a lower rate of adverse events [18], possibly because of still very common use of high-dose corticosteroids. In future studies greater attention will be paid to shortening corticosteroid exposure as is already the case in the PEXIVAS and RITAZAREM studies [19].

\section{Standard Treatment of LN}

With the introduction of corticosteroids and cyclophosphamide, the outcome of patients with systemic lupus erythematosus (SLE), LN, and especially class IV LN considerably improved [20], and 5-year survival of LN increased from $44 \%$ in the period of $1953-1969$ to $82 \%$ in the period of 1990-1995. However, this was also at the expense of severe complications, e.g. sepsis, ischemic heart disease, thrombosis, and avascular bone necrosis [21].

Similarly as in AAV, recent clinical studies dedicated to the induction treatment of LN were aimed at either the reduction of the cumulative dose of cyclophosphamide [22] in white patients with LN or the replacement of cyclophosphamide with mycophenolate mofetil [23, 24].

The Euro-Lupus Nephritis Trial [22] demonstrated that in Caucasian patients with LN, low-dose pulsed cyclophosphamide is as effective as high-dose pulsed cyclophosphamide (cumulative dose of cyclophosphamide: 3 vs. $8.5 \mathrm{~g}$ ). Possibly due to a low number of patients, there was only a statistically nonsignificant trend to a lower rate of severe infections in patients treated with low-dose cyclophosphamide. A recent meta-analysis suggested that there is a really significantly lower risk of infection $(\mathrm{RR}=$ 0.688 ) in patients with LN treated with low-dose compared to high-dose cyclophosphamide [25].

In ALMS (Aspreva Lupus Management Study), mycophenolate was similarly effective as cyclophosphamide in inducing remission of LN [23]. Unfortunately, although the adverse events were partly drug specific (nausea, vomiting, and alopecia were more frequent in cyclophosphamide, and diarrhea was more frequent in mycophenolate-treated subjects), the rate of respiratory and urinary tract infections was the same in both limbs.

An early pre-ALMS meta-analysis comparing induction treatment of LN with mycophenolate versus cyclophosphamide including 847 patients [26] demonstrated that amenorrhea $(R R=0.212)$ and leukopenia $(R R=$ 0.453 ) were significantly less frequent in patients with LN treated with mycophenolate compared to cyclophosphamide. Newer meta-analyses (including ALMS) confirmed the significantly higher risk of amenorrhea $(R R=6.64)$ and alopecia $(R R=5.57)$ in patients treated with cyclophosphamide compared to mycophenolate, but no difference in leukopenia, infections, and gastrointestinal symptoms was demonstrated [27]. Similar results, with the exception of a lower rate of leukopenia (but no difference in infection rate) in patients treated with mycophenolate, were demonstrated in the last published meta-analysis [28].

A recent single-center analysis demonstrated a progressive improvement of 20 -year patient and renal survival and decreased morbidity in patients with LN [29]. 
In patients diagnosed between 1981 and 1990 compared to patients diagnosed between 1968 and 1980, patient survival at 20 years increased from 84 to $95 \%$ and renal survival at 20 years from 75 to $84 \%$, possibly because of earlier referral, but also due to the refinement of treatment accompanied by higher survival without severe infection (66.5 vs. $44 \%$ ) and without cardiovascular events at 20 years (90 vs. $53 \%$ ).

Slow onset of remission (with accumulating chronic damage) and a high rate of relapses requiring reinstitution of effective, but toxic, treatment are the main reasons for the still unsatisfactory long-term outcome of LN [30]; therefore, new and more effective and less toxic modes of treatment with more rapid onset of response are eagerly awaited.

\section{Adverse Events Related to Standard Immunosuppressive Treatment}

Cyclophosphamide may induce nausea and vomiting, bone marrow suppression, alopecia, and hepatotoxicity. Azathioprine may cause myelosuppression and hepatotoxicity and use of methotrexate may result in hepatotoxicity and pulmonary fibrosis. Use of mycophenolate mofetil may be limited by diarrhea. High-dose corticosteroids may induce or worsen hypertension, hyperglycemia and diabetes, weight gain, dyslipidemia, Cushingoid face, striae, fat redistribution, fluid retention, and (less frequently) psychiatric side effects, especially mood disturbances.

Gastrointestinal side effects of cyclophosphamide (nausea and vomiting) definitely occur less frequently in patients with AAV and LN than in patients treated with the same drug (but usually with higher doses) for malignant disease. Complications may be almost completely prevented in patients prone to this by pretreatment with 5-hydroxytryptamine receptor antagonists, e.g. ondansetron.

Infection is definitely the most important side effect of induction treatment of both AAV and LN. The most important long-term adverse events are thromboembolic and cardiovascular events, gonadal dysfunction, malignancy, bone disease, and cataracts. The most frequent self-reported side effect of the treatment in AAV is fatigue (present in a large cross-sectional study in $74.8 \%$ of patients [31] caused by the combination of pain, inflammation, and sleep disturbances).

In the course of both AAV and LN, there is accumulating damage due to both the recurring activity of the disease and side effects of the treatment. In an analysis of long-term follow-up of 467 patients recruited for the EUVAS studies, damage increased during follow-up and the most commonly reported items of treatment-related damage after a mean follow-up of 7.3 years were hypertension (41.5\%), osteoporosis (14.1\%), malignancy (12.6\%), and diabetes (10.4\%) [32]. Similarly, there was damage accrual in a large inception cohort of 1,722 patients with SLE related to the damage at enrolment and predicting future mortality. Steroid use was associated with the transition from no damage to damage, on the other hand use of hydroxychloroquine was associated with a lower rate of increasing damage [33].

\section{Infections}

Infections belong to the most frequent and most dangerous adverse events associated with standard induction immunosuppressive treatment. Infections may also trigger relapses of the disease and may mimic the activity of the disease. Despite great variations in reporting infections, severe infections were very common after the introduction of cyclophosphamide into treatment of AAV with $46 \%$ of patients experiencing severe infection within 8 years of follow-up [4].

Recent analysis of the outcome of 524 patients with AAV recruited to the NORAM, CYCAZAREM, CYCLOPS and MEPEX studies demonstrated that early mortality in the first year of the treatment is more related to therapy-associated adverse events (namely infections related to leukopenia - 59\% of deaths) than to the activity of the disease (only $14 \%$ of deaths) [34], clearly documenting the risks of otherwise effective standard treatment. Infection remains an important cause of death also during the long-term follow-up, being the cause of about $20 \%$ of deaths after the first year of treatment [9]. Although the infection rate in AAV has definitely decreased in the last 20 years $[4,34]$, probably due to the shorter exposure of patients to cyclophosphamide, nonfatal infections still significantly contribute to patient morbidity [35]. The main predictors of infections are leukopenia, intensity and cumulative dose of immunosuppression (both of cyclophosphamide and corticosteroids), and age of the patients [36].

Except for common bacterial infections, patients with AAV may also suffer from severe opportunistic infections, namely Pneumocystis jiroveci infection, which is relatively rare (incidence: $0.85-12 \%$ ), but carries a high 
risk of respiratory failure and mortality in affected patients (47-62.5\%). Infection with $P$. jiroveci may be effectively prevented by trimethoprim/sulfamethoxazole and this treatment is recommended by recent EULAR guidelines [10].

A similarly high infection rate was also reported for patients with lupus $(32-45 \%$ with corticosteroids combined with cyclophosphamide compared to $7-12 \%$ in patients treated with corticosteroids only [18]). Out of 1,000 European patients with SLE, infections occurred in $36 \%$ of them during 10-year follow-up. Infections (25\%) and active disease were also shown to be the most frequent cause of death in the first 5 years after diagnosis in a large European cohort of patients with SLE [37].

In the Euro-Lupus trial [22], severe infections occurred in 15 out of 89 patients (17\% of patients), nominally less frequently in patients treated with low-dose cyclophosphamide. Infections may be related to cyclophosphamide-induced leukopenia, which may occur in $10-30 \%$ of patients with LN (usually after 1-2 weeks after cyclophosphamide administration) and may not be less frequent in patients treated by low-dose cyclophosphamide [22]. In ALMS [23], infections were most frequent among serious adverse events and occurred in $12 \%$ of patients treated by mycophenolate mofetil and $10 \%$ of those treated by cyclophosphamide.

Herpes zoster infection also occurs much more frequently in patients with AAV and LN compared to healthy subjects, and more often in older subjects and patients with decreased renal function [35], although in patients with LN treated with low-dose cyclophosphamide or mycophenolate mofetil, herpes zoster was reported much less frequently than in early NIH studies using high-dose cyclophosphamide [38]. In the EuroLupus study [22], herpes zoster occurred in 7 out of 89 patients (in 5 on high-dose and 2 on low-dose cyclophosphamide).

Infections thus remain the most important cause of morbidity/mortality during the induction treatment of both AAV and LN.

\section{Gonadal Dysfunction}

In SLE, the risk of gonadal dysfunction is related to the age and duration of the treatment with cyclophosphamide. Overall risk of developing menopause with a single course of induction treatment of AAV or LN with cyclophosphamide is generally reported to be lower than $5 \%$, but the cumulative rate of amenorrhea in patients treated

Limitations of Standard

Immunosuppressive Treatment in AAV with cyclophosphamide may be higher (15-27\%) although amenorrhea may be only transient in some patients [18]. High risk of amenorrhea is associated with a cumulative dose of cyclophosphamide of about $20 \mathrm{~g}$ in the women aged $20-30$ years, but the critical cumulative dose of cyclophosphamide may be as low as $5 \mathrm{~g}$ in the age group 40-50 years [35].

Because of higher median age at presentation, the impact on fertility may not be as important in patients with AAV as in patients with SLE; however, gonadal dysfunction may occur quite frequently in premenopausal women with AAV. In early studies in patients treated with high cumulative doses of cyclophosphamide, ovarian failure was reported to occur in $57 \%$ of previously menstruating women [4] and gonadal dysfunction remains frequent even in newer studies with infertility reported in at least $11 \%$ of men and amenorrhea in more than one third of menstruating women [39].

\section{Malignancy}

Increased risk of malignancy in AAV is related namely to the cumulative dose of cyclophosphamide [40]. Long-term risk of malignancy was 1.6 times higher in 535 AAV patients enrolled in 4 randomized controlled trials compared to the general population (possibly higher in patients with GPA compared to patients with microscopic polyangiitis [41]). The risk of bladder cancer, nonmelanoma skin cancer, and acute myeloid leukemia was significantly increased in a Danish cohort of 293 patients with GPA, especially in patients with a cumulative dose of cyclophosphamide exceeding $36 \mathrm{~g}$ [6]. Based on other studies, the risk of skin cancer may be increased even with lower doses of cyclophosphamide. Importantly, there is also a different latency for different types of cancer - slightly more than 1 year for skin cancer, but 6.9-18.5 years for bladder cancer and 7-16 years for acute myeloid leukemia. Increased risk of skin cancer in particular may also be related to the maintenance treatment with azathioprine and methotrexate [42]. No clear risk of cancer preceding GPA [43] lends no support to the idea that cancer and GPA share similar risk factors.

Newer therapeutic regimens aimed at reducing the cumulative dose of cyclophosphamide and the more common use of mesna (binding the cyclophosphamide metabolite acrolein which may cause hemorrhagic cystitis, a risk factor for bladder cancer) could decrease (but not completely eliminate) the risk of secondary malignancies 
in patients treated with cyclophosphamide, but it still remains to be demonstrated. To decrease the risk of cancer, patients with AAV on long-term (and repeated) immunosuppression should stop smoking, avoid sun exposure, and be under long-term surveillance of their physicians and/or dermatologists.

In SLE there is an increased risk of some types of malignancy (especially non-Hodgkin lymphoma, other nonhematological malignancies, lung cancer, and hepatobiliary cancer), which was shown in case of hematological malignancies associated with the exposure to immunosuppressive drugs [44]. A recent meta-analysis of 8 prospective cohorts of SLE patients including approximately 68,000 patients with 401 malignant cases clearly demonstrated a higher risk of hematologic malignancies [standardized incidence ratio $(\mathrm{SIR})=2.9$ ], nonHodgkin lymphoma $(\mathrm{SIR}=5.7)$, leukemia $(\mathrm{SIR}=2.3)$, and myeloma in SLE patients regardless of age, sex, or ethnicity [45]. An increased risk of lymphoma was related to age and male sex, did not seem to be related to the disease activity, and seemed to be related to the cumulative dose of corticosteroids ( $>3.5 \mathrm{~g}$ ) and cyclophosphamide [46].

\section{Thromboembolic Complications}

AAV is associated with increased risk of venous thromboembolism [47]. Compared to a matched healthy population, the risk increases from 0.3 to 1.8 events per 100 patient-years, with risk being highest in the active phase of the disease after disease presentation or during the relapse of the disease, reaching 6.7 events per 100 patientyears [48]. Risk is further increased by therapy, both by (especially high-dose) corticosteroids and cyclophosphamide. Interestingly, even patients with AAV in remission are hypercoagulable with, for example, increased levels of factor VIII (probably due to persistent endothelial activation/dysfunction [49]).

In a multicenter European cohort of 1,000 patients with SLE, thromboses occurred during a follow-up of 10 years in $9.2 \%$ of patients; however, they were the cause of death in $26.1 \%$ of the patients who died $(6.8 \%)$ during follow-up [37], thus demonstrating the very important role of thrombotic events in SLE-related morbidity/mortality. In SLE the risk of thrombosis is much higher in patients with antiphospholipid antibodies, which occurs in about $10 \%$ of patients with SLE and conferred the patients with a $42 \%$ risk of deep vein thrombosis during 20 years of follow-up [50].

\section{Cardiovascular Complications}

Although the increased risk of cardiovascular complications is well established in patients with rheumatoid arthritis and SLE, the disease and treatment-related cardiovascular risk is less well defined in AAV. A recent Danish study [51] suggested that patients with GPA may have significantly increased the risk of hospitalizations for ischemic heart disease and increased risk of acute myocardial infarction $(\mathrm{RR}=2.5)$. Risk of acute myocardial infarction was highest in patients more than 10 years after diagnosis of GPA, males, patients older than 50 years, and patients with a high cumulative dose of cyclophosphamide. Out of 535 patients enrolled in the EUVAS trials with a follow-up of 5 years, $13.8 \%$ experienced at least one cardiovascular event. Older age and diastolic hypertension were independent predictors of cardiovascular events, and the risk of cardiovascular events was significantly lower in patients with positive anti-PR3 antibodies [52].

One study showed cardiovascular disease to be the most common cause of mortality in patients with SLE after more than 5 years of follow-up [37]. Except for traditional risk factors, cardiovascular disease in SLE is related to the presence of antiphospholipid antibodies, activity of the disease (inflammation), and use and cumulative dose of corticosteroids. In a study of 208 SLE patients, the standardized mortality ratio compared to healthy agematched subjects was 2.4 and $48 \%$ of deaths were related to cardiovascular disease [53], with smoking, CRP, and antiphospholipid antibodies being the only independent predictors of cardiovascular mortality. The cumulative dose of prednisone was significantly associated with symptomatic coronary artery disease $(\mathrm{RR}=1.7)$ in a large cohort of SLE patients [54]. In a recent study [55], both the use of corticosteroids and higher corticosteroid dose were predictors of progression of coronary and aortic calcifications (markers of subclinical atherosclerosis).

An apparently higher risk of cardiovascular morbidity/mortality in SLE is further potentiated by treatment with corticosteroids, strongly implicating that especially the chronic use and repeated use of high-dose corticosteroids should be (if possible) avoided.

\section{Bone Disease}

The risk of osteoporosis is increased in AAV due to the activation of proinflammatory cytokines in active disease, decrease of kidney function in a substantial part of the patients, and especially because of treatment. Osteopenia 
was present in at least one place in $57 \%$ and osteoporosis in $21 \%$ of patients with AAV, with a higher overall prevalence of bone disease in postmenopausal women with AAV [56]. Fractures were described to occur in other studies in $1.5-2.5 \%$ of patients with AAV [57]. Cumulative dose of corticosteroids, renal impairment, and low BMI were predictors of reduced bone mass [56]. Corticosteroids negatively impact the trabecular bone and predispose to vertebral, rib, and pelvic fractures; the risk of fractures is related to the daily dose of corticosteroids [58], and the loss of bone is highest at the beginning of the treatment when the doses used are the highest.

In a large European cohort of SLE patients, osteoporosis was diagnosed in $12.1 \%$ during the 10 -year follow-up [37]. Recent studies confirm the presence of osteopenia in $28-46 \%$ and osteoporosis in 3-9\% of SLE patients with fragility fractures in $4.4 \%$ of patients [59]. In a Johns Hopkins cohort of 539 patients with SLE, the cumulative dose of prednisone was significantly associated with the development of osteoporotic fractures $(\mathrm{RR}=2.5)$ [54] and each 2-month exposure to high-dose prednisone led to 1.2fold increase in the avascular bone necrosis. A safe maintenance dose of corticosteroids has never been defined, but a daily dose lower than $7.5 \mathrm{mg}$ of prednisone may limit the corticosteroid-related adverse events [60].

\section{Cataracts and Glaucoma}

Cataracts are common complications of long-term treatment with glucocorticoids. The cumulative dose of corticosteroids was significantly associated with the risk of cataracts $(R R=1.9)$ in a large cohort of SLE patients. Cataracts occurred in $29 \%$ of patients with SLE treated with a mean maintenance daily dose of $5.4 \pm 2.4$ of prednisone for a median period of 75 months [61]. Cataracts were associated with age, disease duration, and cumulative dose of corticosteroids. Glaucoma was much less frequent and occurred in only $3 \%$ of patients (still higher than in the general population). In patients with GPA with a mean duration of corticosteroid treatment of only 39 months, cataracts occurred similarly as in SLE in $28 \%$ of the patients [62].

\section{Diabetes Mellitus}

Diabetes mellitus occurs frequently after high-dose corticosteroid treatment both in AAV and SLE, and there is a substantial burden with diabetes mellitus related to the use of corticosteroids, representing an important risk factor contributing to the high risk of cardiovascular disease in SLE and AAV.

In an inception cohort of 147 patients with AAV treated with corticosteroids and cyclophosphamide, patients receiving corticosteroids beyond 6 months had a marginally significant trend to higher frequency of new-onset diabetes mellitus compared to patients with earlier corticosteroid withdrawal (19\% in patients on no steroids at 6 months and $32 \%$ in patients treated with corticosteroids beyond 6 months, $R R=2.03$ ) [63]. In patients enrolled in an EUVAS studies, diabetes occurred after mean followup of 7.3 years in $10.4 \%$ of patients.

After high-dose corticosteroid treatment, $12.6 \%$ of 127 Korean SLE patients developed diabetes mellitus [64]. Elevated fasting glucose was present in $20.2 \%$ of patients in a large multiethnic cohort of 1,494 patients with SLE [65]. Diabetes mellitus thus occurs frequently in patients with AAV and SLE treated with current corticosteroid regimens, further contributing to their higher cardiovascular risk.

\section{Impact of Treatment on Quality of Life}

Most patients with AAV treated with standard treatment report decreased quality of life, especially in terms of increased fatigue compromising their daily activities [31, 66], but other treatment-related side effects, such as weight gain, Cushingoid face, redistribution of fat, and alopecia (although relatively mild and reversible in most patients), may also have significant impact [39]. Patients with AAV experience a similar quality of life as other patients with chronic diseases, but report substantially lower quality of life compared to the general population, and a high level of fatigue (present in $75 \%$ of patients) seems to be the most important determinant of low quality of life [67]. Fatigue is experienced in as many as $90 \%$ of patients with SLE [68].

In a large survey, $26 \%$ of AAV patients of working age were unemployed, namely due to at least partly modifiable factors as fatigue, depression, disease damage, and being overweight [69]. Similarly, high disability (34\% among patients of working age) is associated with SLE [70].

\section{Reducing Side Effects of the Treatment}

Adverse effects of the treatment may be reduced by early referral to specialized centers so as to start treatment early and prevent unnecessary chronic damage, tai- 
loring the treatment to the severity of the diseases, and minimizing exposure to the treatment which is most often toxic, especially cyclophosphamide and corticosteroids [10].

This can be partly achieved with optimizing the available standard treatment and could be further facilitated with the use of newer (biologic) treatment. Cyclophosphamide pulses should be preferred over oral continuous cyclophosphamide, and the dose of pulsed cyclophosphamide should be reduced in patients with advanced age and decreased glomerular filtration rate $[10,36]$. Doses and intervals between cyclophosphamide pulses should also be modified based on the occurrence and nadir of leukopenia and putative infections should be actively searched.

Some of the adverse effects of treatment may be prevented: prophylaxis with cotrimoxazole may prevent $P$. jirovecii infection, cryopreservation of the sperm and the use of the gonadotropic-releasing hormone analogues may decrease the cyclophosphamide-related gonadotoxicity, screening programs and avoiding smoking may decrease the risk of cyclophosphamide-associated malignancy, the use vitamin $\mathrm{D}$ and calcium supplementation and (in some patients) the administration of bisphosphonates may decrease the risk of corticosteroid-induced bone loss, and prevention with low-molecular-weight heparin should be considered in some AAV patients with a high risk of thromboembolic complications. Vaccination with influenza and pneumococcal vaccines may be recommended before starting (e.g. biologic) treatment, but may be less effective when immunosuppression cannot be delayed.

Prevention of cardiovascular disease includes giving up smoking, regular physical activity, treatment of dyslipidemia and diabetes, and effective control of the activity of the disease (including the use of antimalarials and vitamin D supplementation in SLE) and minimizing exposure to corticosteroids [71]. The benefit of prevention of thrombosis and cardiovascular events with the use of low-dose aspirin, however, remains uncertain both in
AAV [48] and SLE [72]. The data supporting the use of statins specifically in SLE are also scarce and controversial. On one hand, statin use had no effect on the progression of subclinical atherosclerosis (assessed as progression of carotid intima-media thickness) in an unselected group of pubertal SLE patients [73], but, on the other hand, atorvastatin was effective in a subgroup of patients with elevated CRP [74]. Interestingly, statin use was associated with a decrease of SLE activity, but statins had no effect on measured proinflammatory/prothrombotic biomarkers [75].

\section{Conclusions}

Adverse events related to the standard immunosuppressive treatment of AAV and LN are frequent and interfere with the good clinical efficacy of this treatment. Adverse events contribute to the accumulating damage and are an important cause of the high morbidity/mortality of these patients. Except for minimizing the exposure to both cyclophosphamide and high-dose steroids, the search for new corticosteroid-sparing therapeutic strategies, or even better treatment protocols completely avoiding the use of cyclophosphamide and corticosteroids, is clearly warranted. Hopefully the use of these newer drugs will translate to at least similar efficacy and lower longterm toxicity of the immunosuppressive treatment.

\section{Acknowledgements}

Supported by grant No. PRVOUK P25/LF1/2 of the Ministry of Education of the Czech Republic and by grant No. RVO-VFN 64165 of the Ministry of Health of the Czech Republic.

\section{Disclosure Statement}

Dr. Tesar obtained honoraria for lectures from Abbvie, Amgen, Baxter, Boehringer-Ingelheim, GSK and Roche. Dr. Hruskova got a lecture fee from GSK and Roche.

\section{References}

1 Fauci AS, Wolff SM: Wegener's granulomatosis: studies in eighteen patients and a review of the literature. Medicine (Baltimore) 1973; 52:535-561

2 Hollander D, Manning RT: The use of alkylating agents in the treatment of Wegener's granulomatosis. Ann Intern Med 1967;67: 393-398.
- 3 Fauci AS, Haynes BF, Katz P, Wolff SM: Wegener's granulomatosis: prospective clinical and therapeutic experience with 85 patients for 21 years. Ann Intern Med 1983;98:7685.

4 Hoffman GS, Kerr GS, Leavitt RY, Hallahan CW, Lebovics RS, Travis WD, Rottem M, Fauci AS: Wegener granulomatosis: an analy- sis of 158 patients. Ann Intern Med 1992;116: 488-498.

5 Talar-Williams C, Hijazi YM, Walther MM, Linehan WM, Hallahan CW, Lubensky I, Kerr GS, Hoffman GS, Fauci AS, Sneller MC: Cyclophosphamide-induced cystitis and bladder cancer in patients with Wegener granulomatosis. Ann Intern Med 1996;124:477-484. 
6 Faurschou M, Sorensen IJ, Mellemkjaer L, Loft AG, Thomsen BS, Tvede N, Baslund B: Malignancies in Wegener's granulomatosis: incidence and relation to cyclophosphamide therapy in a cohort of 293 patients. J Rheumatol 2008;35:100-105.

7 Mukhtyar C, Flossmann O, Hellmich B, Bacon P, Cid M, Cohen-Tervaert JW, Gross WL, Guillevin L, Jayne D, Mahr A, Merkel PA, Raspe H, Scott D, Wittel J, Yazici H, Luqmani RA; European Vasculitis Study Group (EUVAS): Outcomes from studies of antineutrophil cytoplasm antibody associated vasculitis: a systematic review by the European League against Rheumatism Systemic Vasculitis Task Force. Ann Rheum Dis 2008;67:1004-1010.

8 Holle JU, Gross WL, Latza U, Nölle B, Ambrosch P, Heller M, Fertmann R, ReinholdKeller E: Improved outcome in 445 patients with Wegener's granulomatosis in a German vasculitis center over four decades. Arthritis Rheum 2011;63:257-266.

-9 Flossmann O, Berden A, de Groot K, Hagen C, Harper L, Heijl C, Höglund P, Jayne D, Luqmani R, Mahr A, Mukhtyar C, Pusey C, Rasmussen N, Stegeman C, Walsh M, Westman K; European Vasculitis Study Group: Long-term patient survival in ANCA-associated vasculitis. Ann Rheum Dis 2011;70:488494.

10 Mukhtyar C, Guillevin L, Cid MC, Dasgupta B, de Groot K, Gross W, et al: EULAR recommendations for the management of primary small and medium vessel vasculitis. Ann Rheum Dis 2009;68:310-317.

$\checkmark 11$ Jayne D, Rasmussen N, Andrassy K, Bacon P, Tervaert JW, Dadoniené J, et al: A randomized trial of maintenance therapy for vasculitis associated with antineutrophil cytoplasmic autoantibodies. N Engl J Med 2003;349:3644.

12 De Groot K, Harper L, Jayne DR, Flores Suarez LF, Gregorini G, Gross WL, Luqmani R, Pusey CD, Rasmussen N, Sinico RA, Tesar V, Vanhille P, Westman K, Savage CO; EUVAS (European Vasculitis Study Group): Pulse versus daily oral cyclophosphamide for induction of remission in antineutrophil cytoplasmic antibody-associated vasculitis: a randomized trial. Ann Intern Med 2009;150: 670-680.

13 Walsh M, Flossmann O, Berden A, Westman K, Höglund P, Stegeman C, Jayne D; European Vasculitis Study Group: Risk factors for relapse of antineutrophil cytoplasmic antibodyassociated vasculitis. Arthritis Rheum 2012; 64:542-548.

14 Koldingsnes W, Nossent JC: Baseline features and initial treatment as predictors of remission and relapse in Wegener's granulomatosis. J Rheumatol 2003;30:80-88.

15 Harper L, Morgan MD, Walsh M, Höglund P, Westman K, Flossmann O, Tesar V, Vanhille P, de Groot K, Luqmani R, Flores-Suarez LF, Watts R, Pusey C, Bruchfeld A, Rasmussen N, Blockmans D, Savage CO, Jayne D;
EUVAS Investigators: Pulse versus daily oral cyclophosphamide for induction of remission in ANCA-associated vasculitis: longterm follow-up. Ann Rheum Dis 2012;71: 955-960.

16 Walsh M, Faurschou M, Berden A, Flossmann O, Bajema I, Höglund P, Smith R, Szpirt W, Westman K, Pusey CD, Jayne DR; European Vasculitis Study Group: Long-term follow-up of cyclophosphamide compared with azathioprine for initial maintenance therapy in ANCA-associated vasculitis. Clin J Am Soc Nephrol 2014;9:1571-1576.

17 Faurschou M, Westman K, Rasmussen N, de Groot K, Flossmann O, Höglund P, Jayne DR; European Vasculitis Study Group: Brief report: long-term outcome of a randomized clinical trial comparing methotrexate to cyclophosphamide for remission induction in early systemic antineutrophil cytoplasmic antibody-associated vasculitis. Arthritis Rheum 2012;64:3472-3477.

18 Hogan J, Avasare R, Radhakrishnan J: Is newer safer? Adverse events associated with firstline therapies for ANCA-associated vasculitis and lupus nephritis. Clin J Am Soc Nephrol 2014;9:1657-1667.

19 Walsh M, Merkel PA, Peh CA, Szpirt W, Guillevin L, Pusey CD, De Zoysa J, Ives N, Clark WF, Quillen K, Winters JL, Wheatley K, Jayne D; PEXIVAS Investigators: Plasma exchange and glucocorticoid dosing in the treatment of anti-neutrophil cytoplasm antibody associated vasculitis (PEXIVAS): protocol for a randomized controlled trial. Trials 2013;14:73.

20 Cameron JS: Lupus nephritis. J Am Soc Nephrol 1999;10:413-424.

21 Bono L, Cameron JS, Hicks JA: The very longterm prognosis and complications of lupus nephritis and its treatment. QJM 1999;92: 211-218.

22 Houssiau FA, Vasconcelos C, D’Cruz D, Sebastiani GD, Garrido Ed, Ede R, et al: Immunosuppressive therapy in lupus nephritis: the Euro-Lupus Nephritis Trial, a randomized trial of low-dose versus high-dose intravenous cyclophosphamide. Arthritis Rheum 2002;46:2121-2131.

23 Appel GB, Contreras G, Dooley MA, Ginzler EM, Isenberg D, Jayne D, Li LS, Mysler E, Sánchez-Guerrero J, Solomons N, Wofsy D; Aspreva Lupus Management Study Group: Mycophenolate mofetil versus cyclophosphamide for induction treatment of lupus nephritis. J Am Soc Nephrol 2009;20:11031112.

24 Bertsias GK, Tektonidou M, Amoura Z, Aringer $\mathrm{M}$, Bajema I, Berden $\mathrm{JH}$, et al: Joint European League against Rheumatism and European Renal Association - European Dialysis and Transplant Association (EULAR/ ERA-EDTA) recommendations for the management of adult and paediatric lupus nephritis. Ann Rheum Dis 2012;71:17711782.

25 Lee YH, Woo JH, Choi SJ, Ji JD, Song GG: Induction and maintenance therapy for lupus nephritis: a systematic review and meta-analysis. Lupus 2010;19:703-710.

26 Mak A, Cheak AAC, Tan JAS, Su HC, Ho RC, Lau CS: Mycophenolate mofetil is as efficacious as, but safer than, cyclophosphamide in the treatment of proliferative lupus nephritis: a meta-analysis and meta-regression. Rheumatology 2009;48:944-952.

27 Touma Z, Gladman DD, Urowitz MB, Beyene J, Uleryk EM, Shah PS: Mycophenolate mofetil for induction treatment of lupus nephritis: a systematic review and metaanalysis. J Rheumatol 2011;38:69-78.

28 Liu LL, Jiang Y, Wang LN, Yao L, Li ZL: Efficacy and safety of mycophenolate mofetil versus cyclophosphamide for induction therapy of lupus nephritis: a meta-analysis of randomized controlled trials. Drugs 2012;72: 1521-1533.

29 Moroni G, Quaglini S, Galleli B, Banfi G, Messa $\mathrm{P}$, Ponticelli C: Progressive improvement of patient and renal survival and reduction of morbidity over time in patients with lupus nephritis (LN) followed for 20 years. Lupus 2013;22:810-818.

30 Tesar V, Hruskova Z: Treatment of proliferative lupus nephritis: a slowly changing landscape. Nat Rev Nephrol 2011;7:96109.

31 Basu N, McClean A, Harper L, Amft EN, Dhaun N, Luqmani RA, Little MA, Jayne DR, Flossmann O, McLaren J, Kumar V, Erwig LP, Reid DM, Macfarlane GJ, Jones GT: Explaining fatigue in ANCA-associated vasculitis. Rheumatology (Oxford) 2013;52:16801685.

- 32 Robson J, Doll H, Suppiah R, Flossmann O, Harper L, Höglund P, Jayne D, Mahr A, Westman K, Luqmani R: Damage in the ANCA-associated vasculitides: long-term data from the European Vasculitis Study group (EUVAS) therapeutic trials. Ann Rheum Dis 2013, DOI: 10.1136/annheumdis-2013-203927.

-33 Bruce IN, O’Keefe AG, Farewell V, Hanly JG, Manzi S, Su L, et al: Factors associated with damage accrual in patients with systemic lupus erythematosus: results from the Systemic Lupus International Collaborating Clinics (SLICC) Inception Cohort. Ann Rheum Dis 2014, DOI: 10.1136/annrheumdis-2013-205171.

-34 Little MA, Nightingale P, Verburgh CA, Hauser T, De Groot K, Savage C, Jayne D, Harper L; European Vasculitis Study (EUVAS) Group: Early mortality in systemic vasculitis: relative contribution of adverse events and active vasculitis. Ann Rheum Dis 2010;69:1036-1043.

35 Turnbull J, Harper L: Adverse effects of therapy for ANCA-associated vasculitis. Best Pract Res Clin Rheumatol 2009;23:391401.

36 Harper L, Savage CO: ANCA-associated renal vasculitis at the end of the twentieth century a disease of older patients. Rheumatology (Oxford) 2005;44:495-501. 
- 37 Cervera R, Khamashta MA, Font J, Sebastiani GD, Gil A, Lavilla P, et al: Morbidity and mortality in systemic lupus erythematosus during a 10-year period: a comparison of early and late manifestations in a cohort of 1,000 patients. Medicine (Baltimore) 2003; 82:299-308.

38 Felson DT, Anderson J: Evidence for the superiority of immunosuppressive drugs and prednisone over prednisone alone in lupus nephritis. Results of a pooled analysis. N Engl J Med 1984;311:1528-1533.

- 39 Seo P, Min YI, Holbrook JT, Hoffman GS, Merkel PA, Spiera R, Davis JC, Ytterberg SR, St Clair EW, McCune WJ, Specks U, Allen NB, Luqmani RA, Stone JH; WGET Research Group: Damage caused by Wegener's granulomatosis and its treatment: prospective data from the Wegener's Granulomatosis Etanercept Trial (WGET). Arthritis Rheum 2005;52: 2168-2178.

-40 Mahr A, Heijl C, Le Guenno G, Faurschou M: ANCA-associated vasculitis and malignancy: current evidence for cause and consequence relationships. Best Pract Res Clin Rheumatol 2013;27:45-56.

-41 Heijl C, Harper L, Flossmann O, Stücker I, Scott DG, Watts RA, Höglund P, Westman K, Mahr A; European Vasculitis Study Group (EUVAS): Incidence of malignancy in patients treated for antineutrophil cytoplasm antibody-associated vasculitis. Follow-up data from European Vasculitis Study Group clinical trials. Ann Rheum Dis 2011;70:14151421.

42 Westman KW, Bygren PG, Olsson H, Ranstam J, Wieslander J: Relapse rate, renal survival, and cancer morbidity in patients with Wegener's granulomatosis or microscopic polyangiitis with renal involvement. J Am Soc Nephrol 1998;9:842-852.

43 Faurschou M, Mellemkjaer L, Sorensen IJ, Thomsen BS, Dreyer L, Baslund B: Cancer preceding Wegener's granulomatosis: a casecontrol study. Rheumatology (Oxford) 2009; 48:421-424.

44 Bernatsky S, Joseph L, Boivin JF, Gordon C, Urowitz M, Gladman D, et al: The relationship between cancer and medication exposures in systemic lupus erythematosuus: a case-cohort study. Ann Rheum Dis 2008;67: 74-79.

45 Apor E, O’Brien J, Stephen M, Castillo JJ: Systemic lupus erythematosus is associated with increased incidence of hematologic malignancies: a meta-analysis of prospective cohort studies. Leuk Res 2014;38:1067-1071.

-46 Bernatsky S, Ramsey-Goldman R, Labrecque J, Joseph L, Boivin JF, Petri M, Zoma A, et al: Cancer risk in systemic lupus: and updated international multi-centre cohort study. J Autoimmun 2013;42:130-135.

-47 Merkel PA, Lo GH, Holbrook JT, Tibbs AK, Allen NB, Davis JC Jr, Hoffman GS, McCune WJ, St Clair EW, Specks U, Spiera R, Petri M, Stone JH; Wegener's Granulomatosis Etanercept Trial Research Group: High incidence of venous thrombotic events among patients with Wegener granulomatosis: the Wegener's Clinical Occurrence of Thrombosis (WeCLOT) Study. Ann Intern Med 2005; 142:620-626.

48 Stassen PM, Derks RP, Kallenberg CG, Stegeman CA: Venous thromboembolism in ANCA-associated vasculitis - incidence and risk factors. Rheumatology (Oxford) 2008;47: 530-534.

49 Hilhorst M, Winckers K, Wilde B, van Oerle $\mathrm{R}$, ten Cate H, Tervaert JW: Patients with antineutrophil cytoplasmic antibodies associated vasculitis in remission are hypercoagulable. J Rheumatol 2013;40:2042-2046.

50 Somers E, Magder LS, Petri M: Antiphospholipid antibodies and incidence of venous thrombosis in a cohort of patients with systemic lupus erythematosus. J Rheumatol 2002;29:2531-2536.

51 Faurschou M, Mellemkjaer L, Sorensen IJ, Svalgaard Thomsen B, Dreyer L, Baslund B: Increased morbidity from ischemic heart disease in patients with Wegener's granulomatosis. Arthritis Rheum 2009;60:1187-1192.

52 Suppiah R, Judge A, Batra R, Flossmann O, Harper L, Höglund P, Javaid MK, Jayne D, Mukhtyar C, Westman K, Davis JC Jr, Hoffman GS, McCune WJ, Merkel PA, St Clair EW, Seo P, Spiera R, Stone JH, Luqmani R: A model to predict cardiovascular events in patients with newly diagnosed Wegener's granulomatosis and microscopic polyangiitis. Arthritis Care Res (Hoboken) 2011;63: 588-596.

53 Gustafsson JT, Simard JF, Gunnarsson I, Elvin K, Lundberg IE, Hansson LO, Larsson A, Svenungsson E: Risk factors for cardiovascular mortality in patients with systemic lupus erythematosus, a prospective cohort study. Arthritis Res Ther 2012;14:R46.

54 Zonana-Nacach A, Barr SG, Magder LS, Petri $\mathrm{M}$ : Damage in systemic lupus erythematosus and its association with corticosteroids. Arthritis Rheum 2000;43:1801-1808.

55 Lertratanakul A, Wu A, Dyer AR, Kondos G, Edmundowicz D, Carr J, Ramsey-Goldman R: Risk factors in the progression of subclinical atherosclerosis in women with systemic lupus erythematosus. Arthritis Care Res (Hoboken) 2014;66:1177-1185.

56 Boomsma MM, Stegeman CA, Kramer AB, Karsijns M, Piers DA, Tervaert JW: Prevalence of reduced bone mineral density in patients with anti-neutrophil cytoplasmic antibody associated vasculitis and the role of immunosuppressive therapy: a cross-sectional study. Osteoporos Int 2002;13:74-82.

57 Guillevin L, Cohen P, Mahr A, Arène JP, Mouthon L, Puéchal X, Pertuiset E, Gilson B, Hamidou M, Lanoux P, Bruet A, Ruivard M, Vanhille P, Cordier JF: Treatment of polyarteritis nodosa and microscopic polyangiitis with poor prognosis factors: a prospective trial comparing corticosteroids and six or twelve cyclophopshamide pulses in sixty-five patients. Arthritis Rheum 2003;49:93-100.
58 Van Staa TP, Leufkens HG, Abenhaim L, Zhang B, Cooper C: Oral corticosteroids and fracture risk: relationship to daily and cumulative doses. Rheumatology (Oxford) 2000;39: 1383-1389.

59 Salman-Monte TC, Torente-Segarra V, Muñoz-Ortego J, Mojal S, Carbonell-Abelló J: Prevalence and predictors of low bone density and fragility fractures in women with systemic lupus erythematosus in a Mediterranean region. Rheumatol Int 2014, Epub ahead of print.

60 Ruiz-Irastorza G, Danza A, Khamashta M: Glucocorticoid use and abuse in SLE. Rheumatology (Oxford) 2012;51:1145-1153.

61 Carli L, Tani C, Querci F, Della Rossa A, Vagnani S, Baldini C, Talarico R, d'Ascanio A, Neri R, Tavoni AG, Bombardieri S, Mosca M: Analysis of the prevalence of cataracts and glaucoma in systemic lupus erythematosus and evaluation of the rheumatologist's practice for the monitoring of glucocorticoid eye toxicity. Clin Rheumatol 2013;32:1071-1073.

62 Kamali S, Erer B, Artim-Esen B, Gul A, Ocal L, Konice M, Aral O, Inanc M: Predictors of damage and survival in patients with Wegener's granulomatosis: analysis of 50 patients. J Rheumatol 2010;37:374-378.

63 McGregor JG, Hogan SL, Hu Y, Jennette CE, Falk RJ, Nachman PH: Glucocorticoids and relapse and infection rates in anti-neutrophil cytoplasmic antibody disease. Clin J Am Soc Nephrol 2012;7:240-247.

$64 \mathrm{Ha}$ Y, Lee KH, Jung S, Lee SW, Lee SK, Park YB: Glucocorticoid-induced diabetes mellitus in patients with systemic lupus erythematosus treated with high-dose glucocorticoid therapy. Lupus 2011;20:1027-1034.

65 Parker B, Urowitz MB, Gladman DD, Lunt M, Donn R, Bae SC, et al: Clinical associations of the metabolic syndrome in systemic lupus erythematosus: data from an international inception cohort. Ann Rheum Dis 2013;72: 1308-1314.

66 Hoffman GS, Drucker Y, Cotch MF, Locker GA, Easley K, Kwoh K: Wegener's granulomatosis: patient-reported effects of disease on health, function, and income. Arthritis Rheum 1998;41:2257-2262.

- 67 Basu N, McClean A, Harper L, Amft EN, Dhaun N, Luqmani RA, Little MA, Jayne DR, Flossmann O, McLaren J, Kumar V, Erwig LP, Reid DM, Jones GT, Macfarlane GJ: The characterisation and determinants of quality of life in ANCA associated vasculitis. Ann Rheum Dis 2014;73:207-211.

68 Fonseca R, Bernardes M, Terroso G, de Sousa $M$, Figueiredo-Braga M: Silent burdens in disease: fatigue and depression in SLE. Autoimmune Dis 2014;2014:790724.

69 Basu N, McClean A, Harper L, Amft EN, Dhaun N, Luqmani RA, Little MA, Jayne DR, Flossmann O, McLaren J, Kumar V, Erwig LP, Reid DM, Macfarlane GJ, Jones GT: Markers for work disability in anti-neutrophil cytoplasmic antibody-associated vasculitis. Rheumatology (Oxford) 2014;53:953-956. 
70 Baker K, Pope J: Employment and work disability in systemic lupus erythematosus. Rheumatology (Oxford) 2009;48:281-284.

-71 Iaccarino L, Bettio S, Zen M, Nalotto L, Gatto M, Ramonda R, Punzi L, Doria A: Premature coronary heart disease in SLE: can we prevent progression? Lupus 2013;22:1232-1242.

72 Arnaud L, Mathian A, Ruffatti A, Erkan D, Tektonidou M, Cervera R, Forastiero R, Pengo $\mathrm{V}$, Lambert M, Martinez-Zamora MA, Balasch J, Zuily S, Wahl D, Amoura Z: Efficacy of aspirin for the primary prevention of throm- bosis in patients with antiphospholipid antibodies: an international and collaborative meta-analysis. Autoimmun Rev 2014;13:281291.

73 Schanberg LE, Sandborg C, Barnhart HX, Ardoin SP, Yow E, Evans GW, et al: Use of atorvastatin in systemic lupus erythematosus in children and adolescents. Arthritis Rheum 2012;64:285-296.

74 Ardoin SP, Schanberg LE, Sandborg CI, Barnhart HX, Evans GW, Yow E, et al: Secondary analysis of APPLE study suggests atorvastatin may reduce atherosclerosis progression in pubertal lupus patients with higher $\mathrm{C}$ reactive protein. Ann Rheum Dis 2014;73:557-566.

75 Willis R, Seif AM, McGwin G Jr, MartinezMartinez LA, González EB, Doan E, Dang N, Papalardo E, Liu J, Vilá LM, Reveille JD, Alarcón GS, Pierangeli SS: Effects of statins on proinflammatory/prothrombotic biomarkers and on disease activity scores in SLE patients: data from LUMIN (LXXVI), a multi-ethnic US cohort. Clin Exp Rheumatol 2014;32:162167. 